\title{
Un géant de l'agro-business dans le Mato Grosso
}

Um gigante do agronegócio em Mato Grosso

A giant of agribusiness in Mato Grosso

Un gigante del agronegocio en Mato Grosso

\section{Hervé Théry}

\section{OpenEdition}

\section{Journals}

Édition électronique

URL : http://journals.openedition.org/espacoeconomia/1256

DOI : 10.4000/espacoeconomia.1256

ISSN : 2317-7837

Éditeur

Núcleo de Pesquisa Espaço \& Economia

\section{Référence électronique}

Hervé Théry, « Un géant de l'agro-business dans le Mato Grosso », Espaço e Economia [En ligne], 5 | 2014, mis en ligne le 26 décembre 2014, consulté le 10 juin 2020. URL : http://

journals.openedition.org/espacoeconomia/1256 ; DOI : https://doi.org/10.4000/espacoeconomia. 1256

Ce document a été généré automatiquement le 10 juin 2020.

C N NUPEE 


\title{
Un géant de l'agro-business dans le Mato Grosso
}

\author{
Um gigante do agronegócio em Mato Grosso \\ A giant of agribusiness in Mato Grosso \\ Un gigante del agronegocio en Mato Grosso
}

Hervé Théry

\section{NOTE DE L'AUTEUR}

Une vidéo institutionnelle de $15 \mathrm{mn}$ est disponible sur le site du groupe <http:// www.bomfuturo.com.br/>.

\section{Un géant de l'agro-business dans le Mato Grosso}

1 On dit ici et là que le Brésil est devenu «la ferme du monde », comme la Chine est devenue sont usine et l'Inde son bureau. De fait, il a le rare privilège de disposer de millions d'hectares de terres arables disponibles, qui sont progressivement mises en culture para un puissant complexe agro-industriel. Déjà premier producteur mondial de café et de sucre, premier exportateur mondial de viande de bœuf et de jus d'orange concentré, il est aussi plus récemment devenu le second producteur mondial de soja.

2 Cette montée en puissance est en grande partie due à la mise en place dans les régions pionnières du Centre-Ouest, principalement dans le Mato Grosso, d'immenses domaines agro-pastoraux comme celui du groupe Bom Futuro, situé à Campo Verde, à une centaine de kilomètres à l'Est de Cuiabá, la capitale du Mato Grosso (figures $n^{\circ} 1$ et 2). Un voyage récent m'a permis de le visiter pour la deuxième fois et les informations cidessous, sans constituer un scoop, apportent je crois quelques éléments concrets pour faire comprendre l'immensité de ces nouvelles exploitations agricoles. 
Figure $\mathrm{n}^{\circ} 1$ Localisation du Mato Grosso

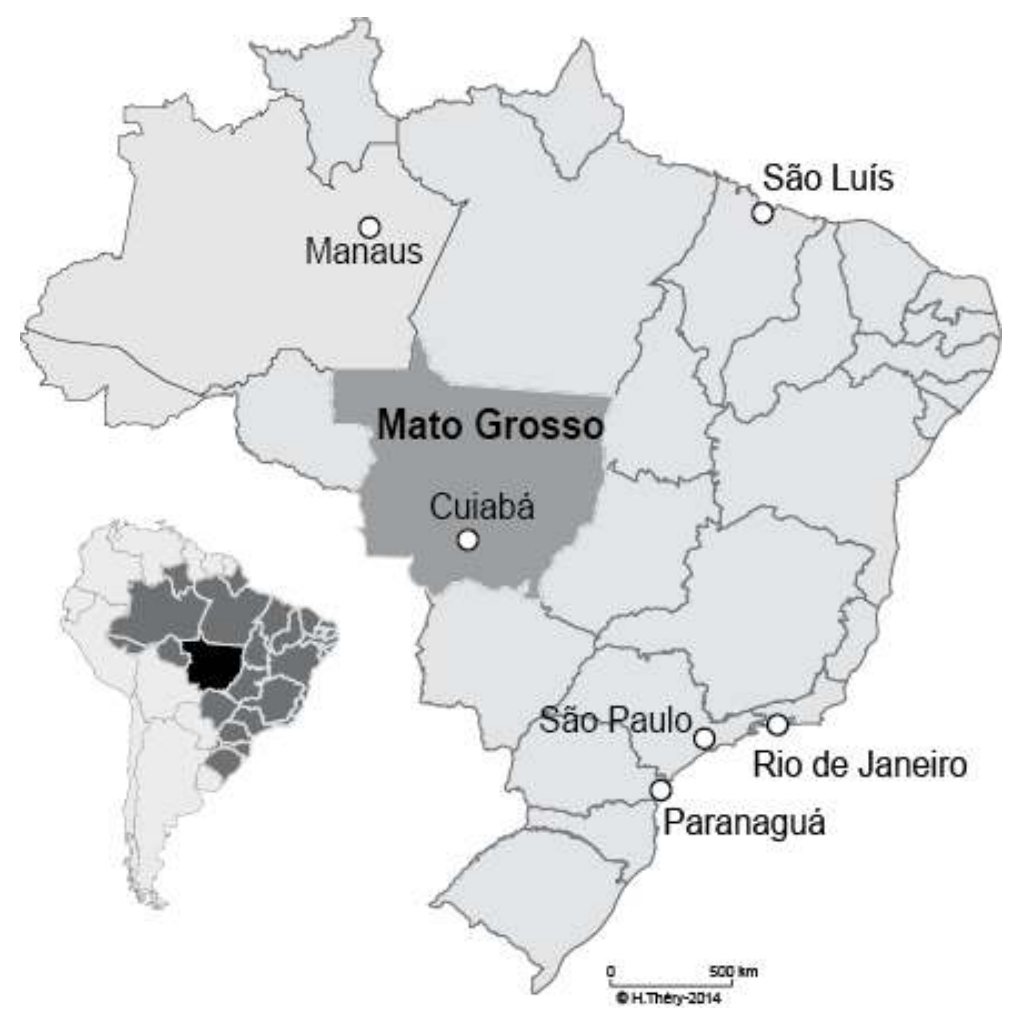

\section{L'entreprise}

Le groupe ${ }^{1}$ appartient à la famille Maggi-Scheffer, ses propriétaires sont trois frères et un beau-frère, qui ont commencé leurs activités agricoles en 1964 à São Miguel do Iguaçu, dans le Paraná. En 1982 ils se sont installés dans le Mato Grosso, alors au début de son boom agricole. En 1988 ils cultivaient 3000 ha en partenariat avec d'autres membres de la famille Maggi, dont l'un des membres, a été un temps le plus grand producteur de soja au monde, et gouverneur du Mato Grosso. En 1993 les quatre associés prennent leur indépendance, achètent la fazenda Bom Futuro et en 1994 ils commencent à y planter du coton.

Ce choix sera une des raisons principales de leur succès, avec la pratique systématique d'une deuxième récolte annuelle (soja + coton ou soja + maïs), le semis direct dans les chaumes (sans labour) et la diversification (élevage, pisciculture, production de semences). 
Figure $n^{\circ} 2$ Localisation du groupe Bom Futuro

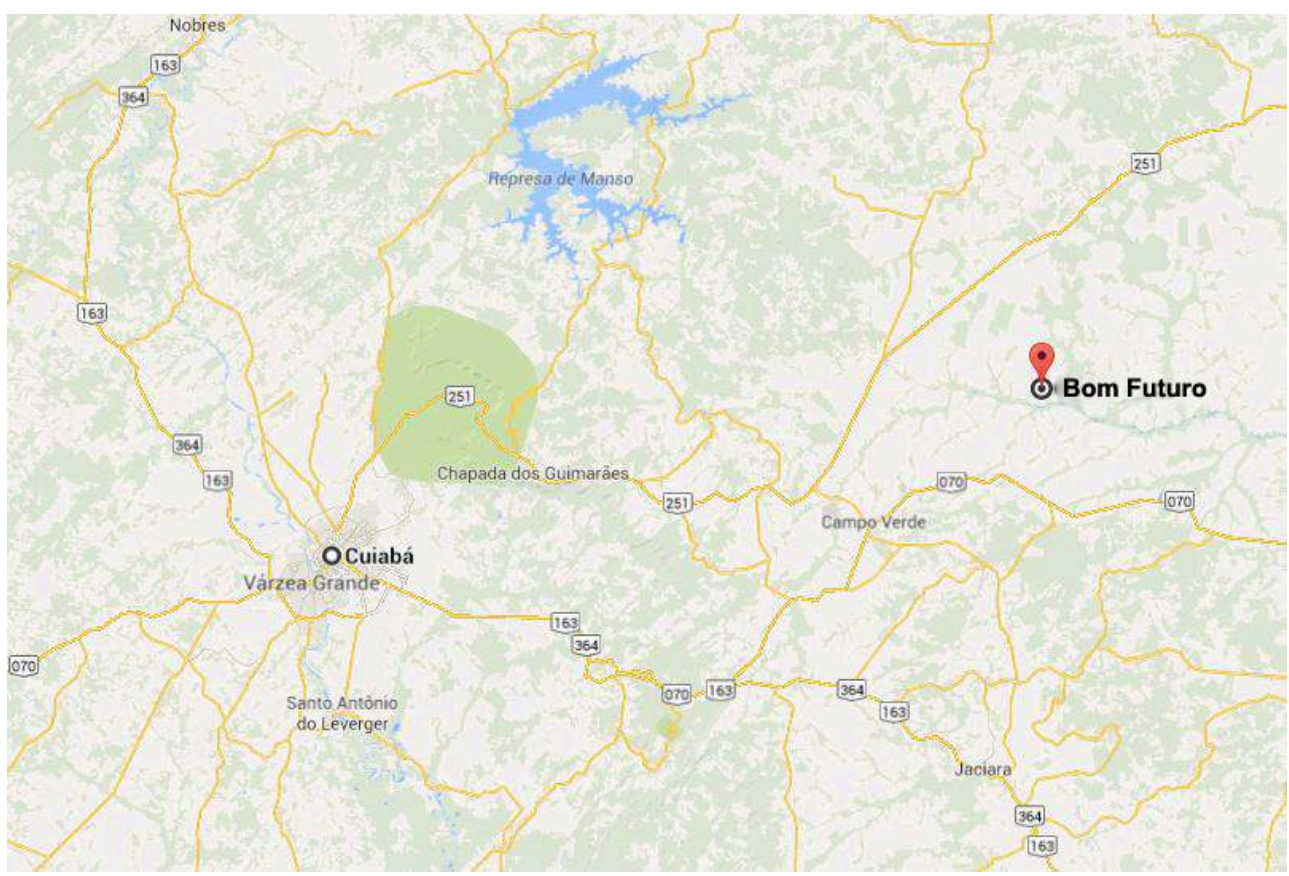

\section{Les dimensions}

5 À sa fondation, le groupe cultivait 2500 ha. Aujourd'hui il en cultive 420000 (en deux récoltes, sur 250000 cultivables), et le domaine compte en outre 160000 de terres destinées à l'élevage et aux réserves forestières que la loi brésilienne leur fait obligation de conserver.

\begin{tabular}{lr} 
Soja: & $230000 \mathrm{ha}$ \\
Mais: & $105000 \mathrm{ha}$ \\
Coton: & $75000 \mathrm{ha}$ \\
Autres cultures: & $10000 \mathrm{ha}$ \\
\hline Total: & $420000 \mathrm{ha}$
\end{tabular}

Toutes ces terres n'appartiennent pas du groupe, la moitié sont louées à des propriétaires qui ont chacun 1000 ou 2000 ha. Le prix de la location est calculé en sacs de soja, l'unité de compte universelle dans la région, coté à 57,65 Reais le 21 février 2014 à Primavera do Leste - la référence locale - soit 17,74 Euros à la même date. En moyenne elle se monte à 9 sacs par ha et par an (160 Euros, mais variant de 5 sacs pour des terres sableuses sans infrastructure à 13 sacs pour des terres argileuses et dotées de bonnes infrastructures, comme des silos, l'électricité, etc.). À l'achat ces terres vaudraient de 80 à 100 sacs (1 400 à 1800 Euros) pour les pour les terres sableuses à 400-500 (7 000 à 9000 Euros) pour des terres argileuses proches de la ville. Il y a 10 ans les prix moyens étaient respectivement de 50 et 200 sacs (900 et 3500 Euros au cours actuel du soja et des monnaies), ils ont beaucoup monté avec les prix des commodities, les denrées cotées en bourse et demandées par le marché international, la Chine en tête. 
7 Le groupe de souhaite pas s'agrandir davantage, mais ses propriétaires saisissent les occasions qui se présentent et cherchent à diversifier, sans renoncer à la sécurité climatique que leur offre le climat du Mato Grosso. Récemment ils ont acheté des terres dans la vallée de l'Araguaia, plus à l'Est, visant l'exportation de leur production par un chemin de fer, encore en construction, qui permettra une sortie par le port de São Luis, dans le Maranhão. En outre, en réponse aux nouvelles règles environnementales qui ont augmenté la proportion de forêts à conserver, ils cherchent à acheter des terres forestières, dans la même commune ou au moins dans le même bassin fluvial, comme le prescrit la loi.

Figure $\mathrm{n}^{\circ} 3$ Les terres du groupe Bom Futuro

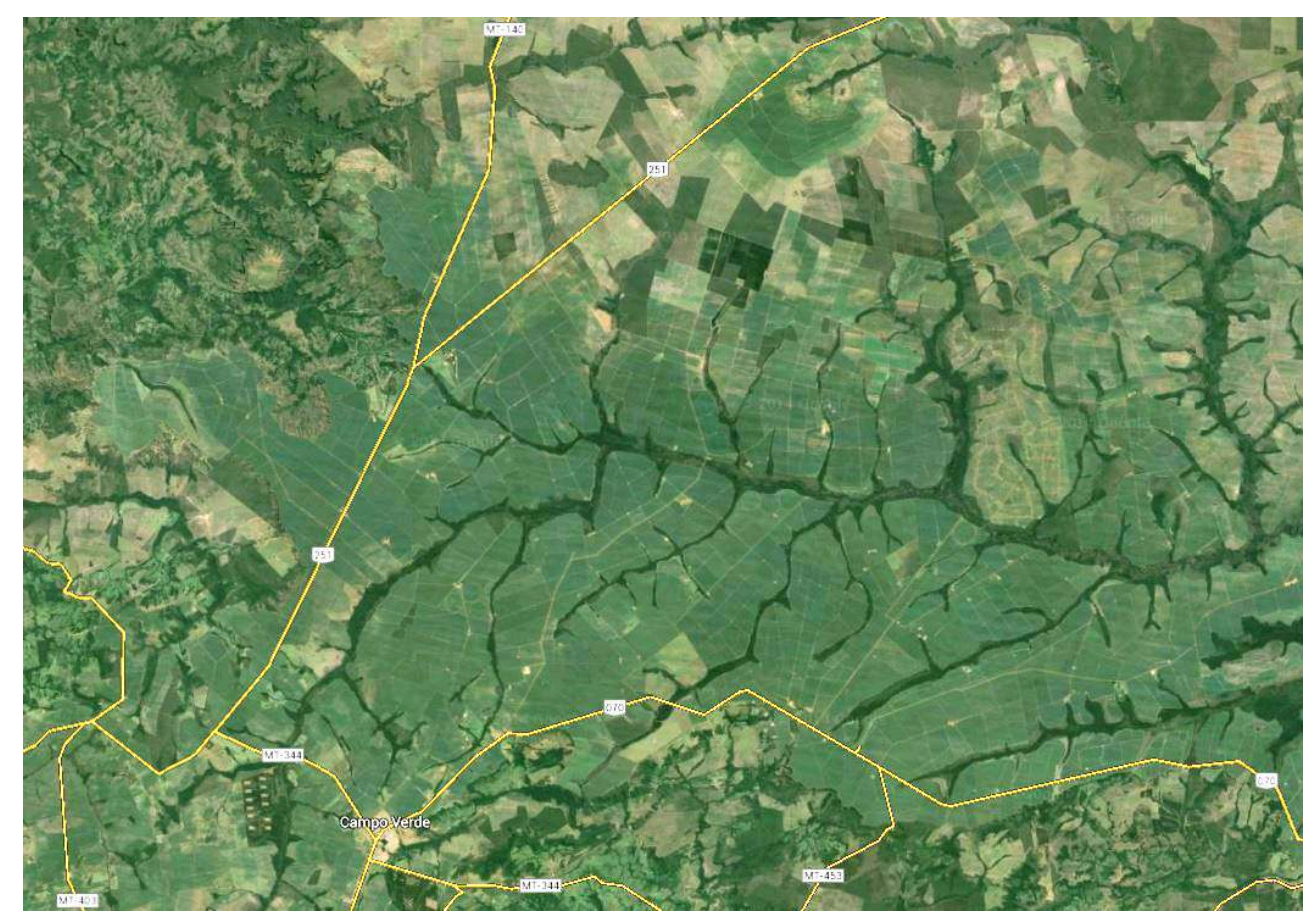


Figure $\mathrm{n}^{\circ} 4$ Le siège principal, situé à 32 kilomètres de l'entrée.

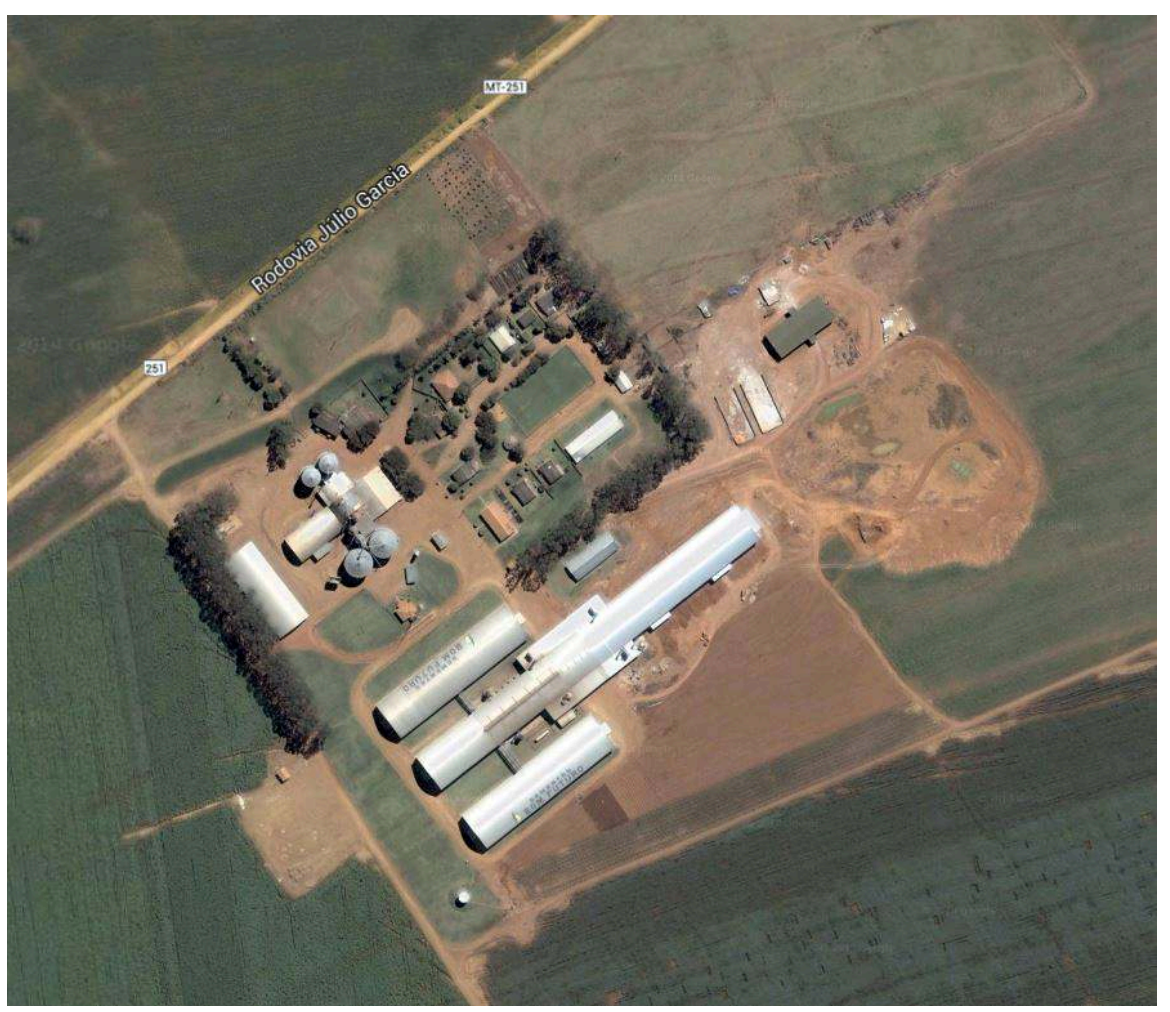

Figure $\mathrm{n}^{\circ} 5$ Une des batteries de silos pour le stockage des grains

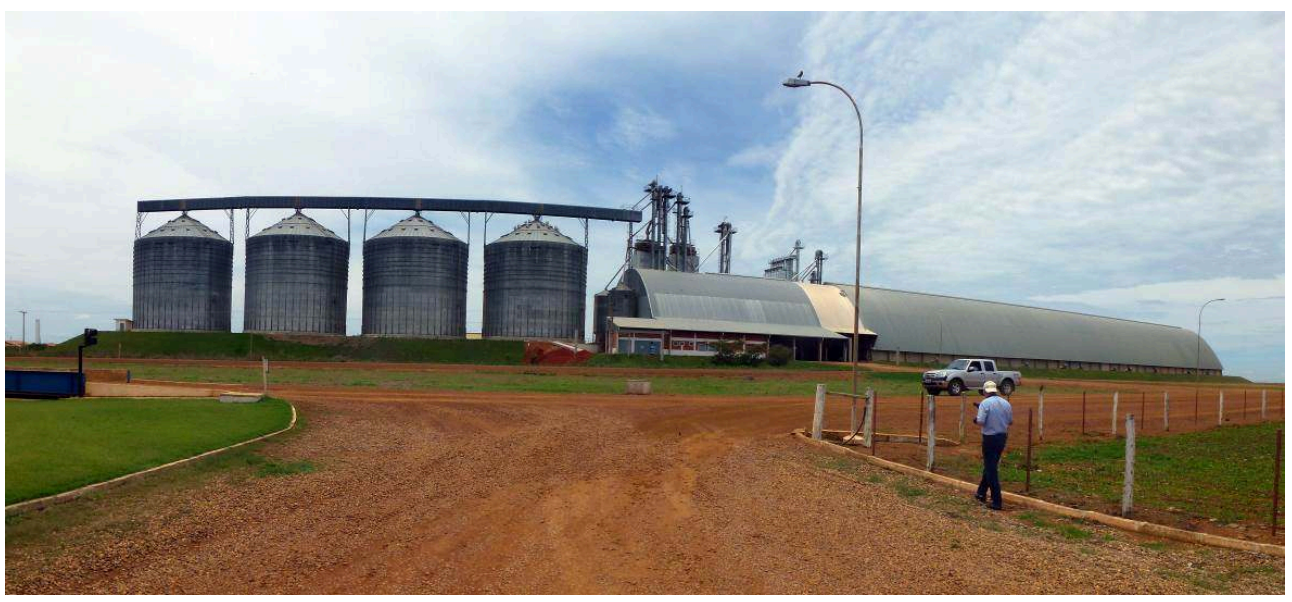

\section{La production agricole}

Le rendement en soja est en moyenne de 53 sacs par hectare, soit 3,2 $t$, mais cette année 2014 le groupe attend, si tout se passe bien, un rendement de $3,5 \mathrm{t} / \mathrm{ha}$, avec de bon prix. Le maïs produit $6 \mathrm{t} /$ ha en moyenne, comme il est planté en deuxième culture, il dépend beaucoup de la quantité de précipitations en fin de saison des pluies. C'est une loterie, mais qui peut donner de très bon résultats et de toute façon coûte peu, juste peu d'engrais puisqu'il utilise les mêmes terres, machines, silos et personnels que le soja qui le précède. Il est normalement prêt en 115 à 125 jours, mais souvent récolté à 140-150 
jours puisqu'il clôt le cycle annuel et peut rester en terre sans dommage en période sèche.

9 À la fondation du groupe, le coton rapportait - selon le directeur technique - sept fois plus que le soja, mais cette différence tend à s'estomper, et ses cotations fluctuent beaucoup selon les années. Le marché est assuré puisque le Brésil consomme $800000 \mathrm{t}$ de fibres par an, et exporte le reste, mais pas tellement vers la Chine, un marché conquis par USA. En outre, comme le Brésil a réussi à conserver une partie de son industrie textile, les prix internes sont parfois meilleurs que ceux de l'exportation.

Outre le coton, un autre facteur qui a permis le succès de l'entreprise a été l'adoption du "semis direct", qui consiste a planter une récolte dans les chaumes de la précédente, sans labours ni hersage. Expérimentée dans les années 1980 - avec l'aide du Cirad, l'organisme français de coopération agricole, cette pratique a permis entre autres avantages de réduire l'érosion des sols - qui ne restent découverts que quelques jours, d'augmenter la teneur en matière organique des sols et les rendements.

11 Le coton ou le maïs sont donc semés juste après la récolte du soja, ce qui donne le temps d'une seconde récolte sur les meilleures terres, sur les plus sableuses on sème plutôt un millet destiné à devenir un engrais vert ou de la brachiara (Brachiaria brizantha) une graminée fourragère particulièrement utile pour son pouvoir restructurant sur les sols.

Figure $\mathrm{n}^{\circ} 6$ Semis direct dans la culture d'engrais vert

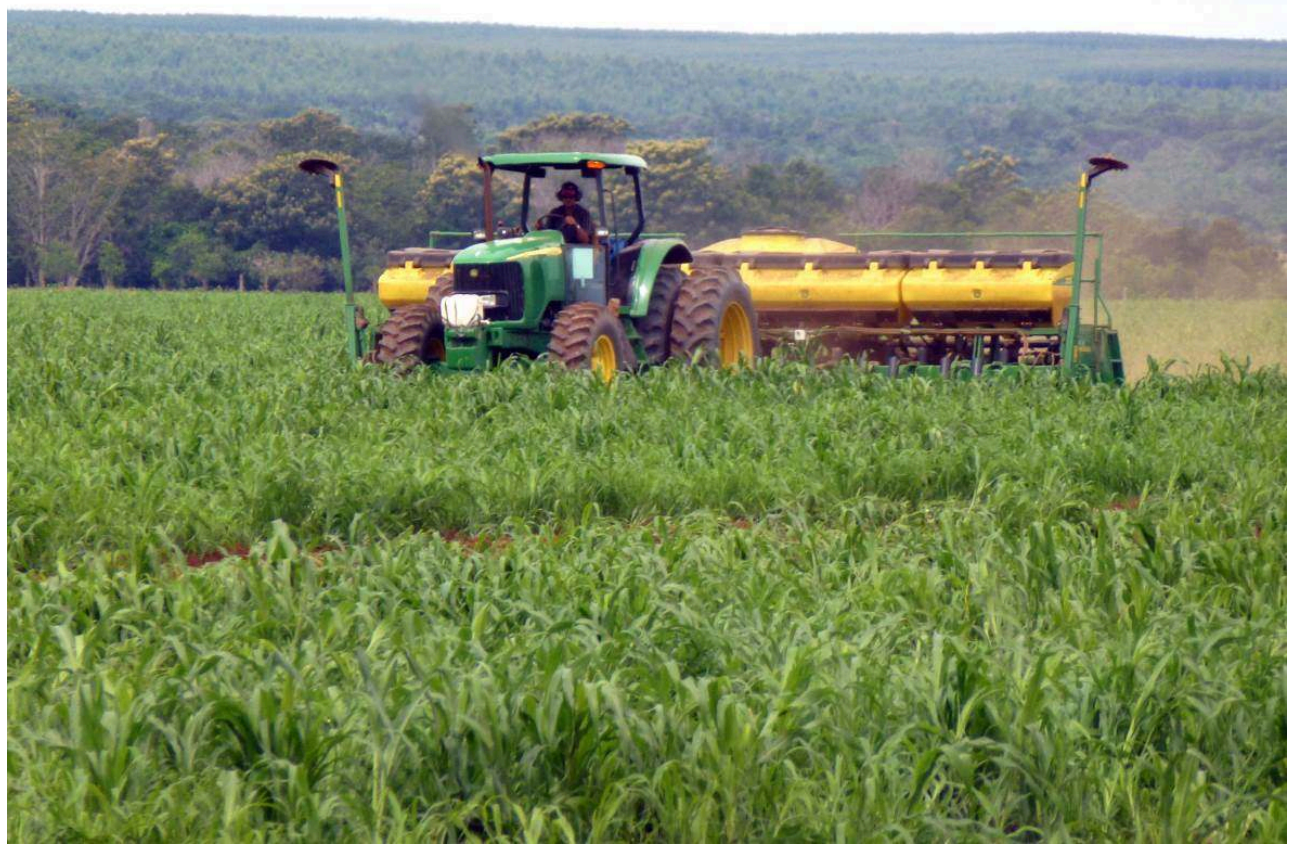

12 Tout cela suppose évidemment une mécanisation massive et les machines en tout genre ne manquent pas dans les fazendas du groupe, depuis les semoirs capables de semer des grains sur près de cinquante rangs à la fois jusqu'aux moissonneuses-batteuses dont les barres de coupe ont une envergure de plus de quinze mètres. 
Figure $n^{\circ} 7$ Un semoir à 49 lignes

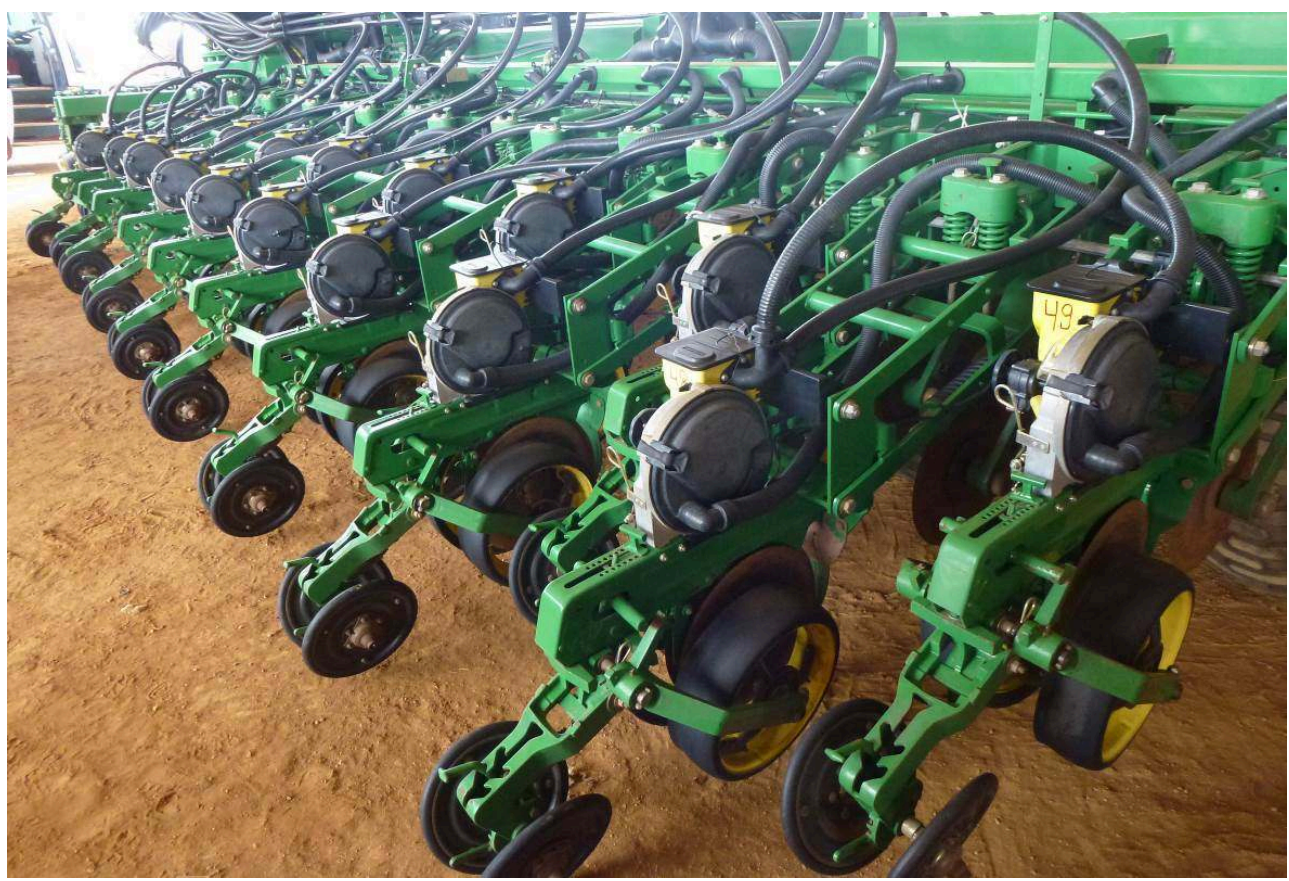

13 Au moment des récoltes, et tout particulièrement de celle du soja, qui était en cours lors de notre visite en février 2014, s'organise donc un dans les champs et sur les routes un ballet complexe de moissonneuses, transbordeurs, camions de transport vers les silos, tracteurs et semoirs, camions citernes, etc.

Figure $n^{\circ} 8$ Récolte du soja à la fazenda Filadelfia

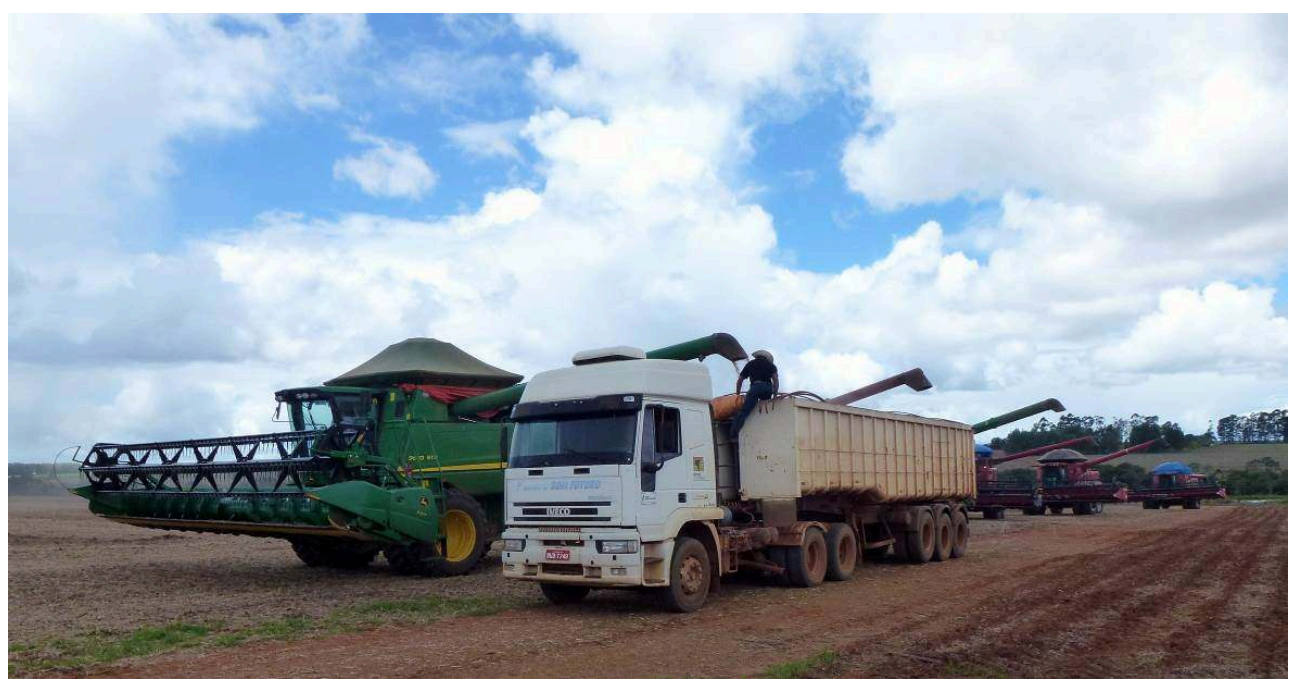


Figure $n^{\circ} 9$ Une des moissonneuses-batteuses

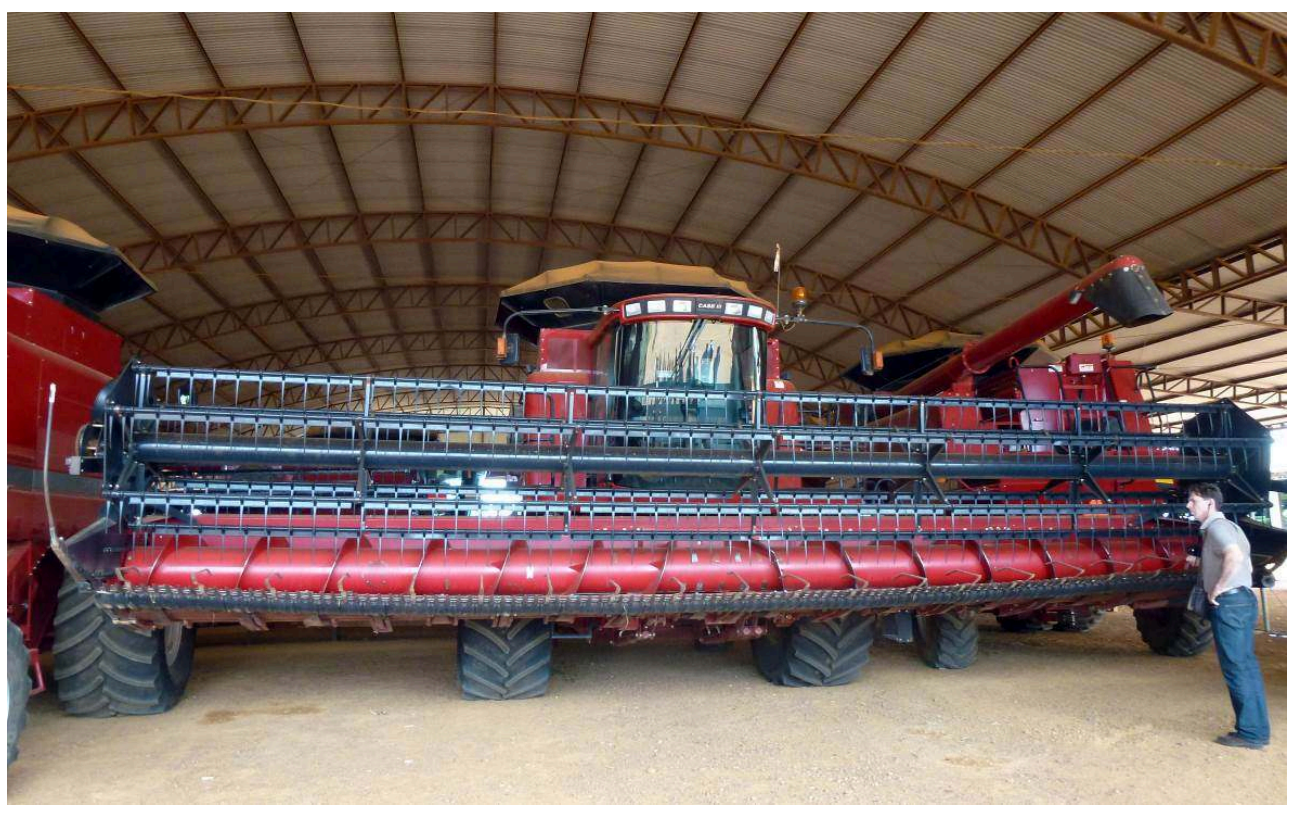

Au moment de la récolte il faut en outre traiter avec un soin particulier les parcelles destinées aux semences de la campagne suivante. La partie du domaine dont c'est la spécialité produit plus de 700000 sacs de $60 \mathrm{~kg}$ de graines de soja par an (la livraison des semences se fait dans de grands bacs, mais ici aussi la production est comptée en sacs). Bom Futuro en utilise environ $30 \%$ (et vend le reste) et produit également ses semences de coton, de maïs, de sorgho et d'autres cultures.

Dans d'autres parties du domaine des installations de type plus industriel qu'agricole sont destinées au traitement du coton qui est expédié en fardeaux comprimés vers les filatures ou les ports d'embarquement.

\section{Figure $n^{\circ} 10$ Expédition des fardeaux de coton}

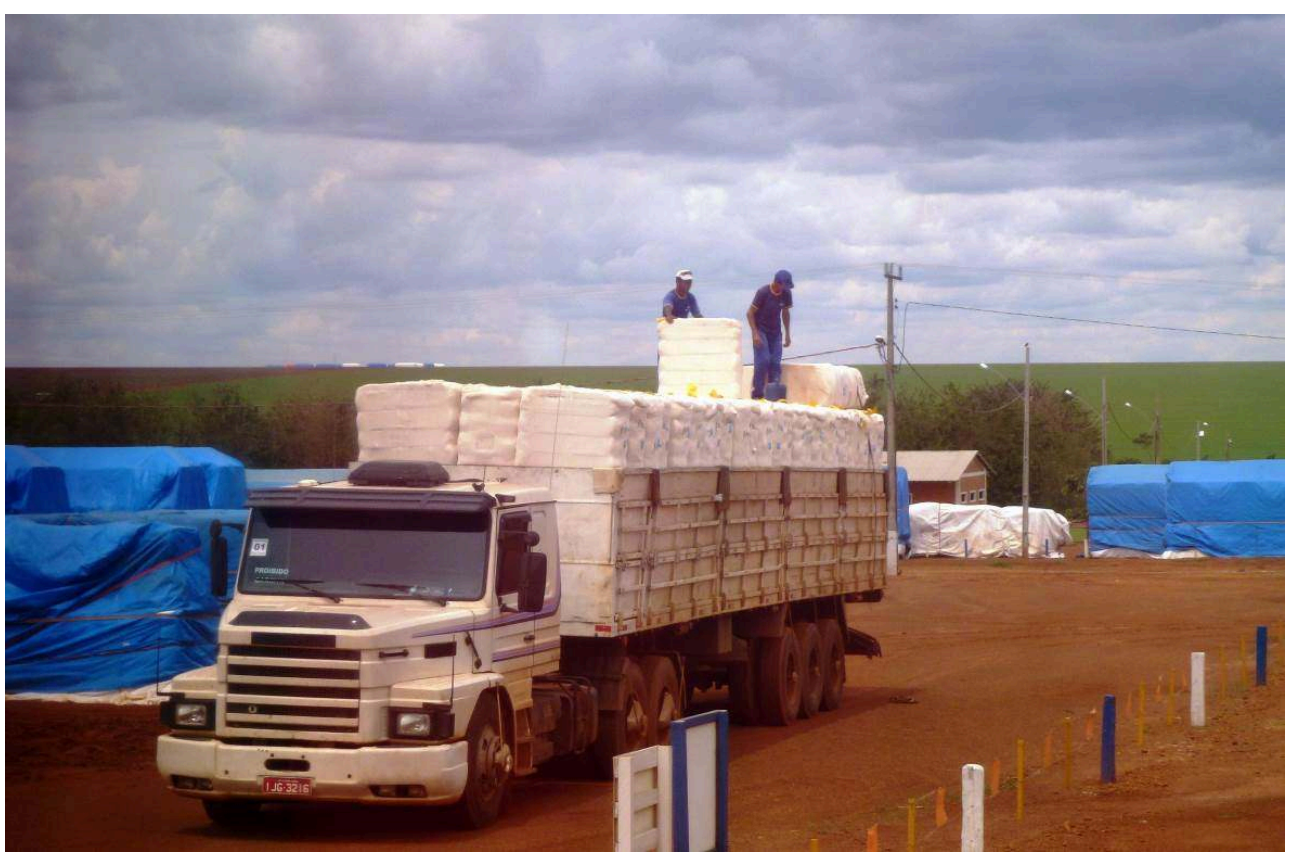




\section{Pisciculture}

À côté de ces activités agricoles, le domaine comprend une partie consacrée à la pisciculture, avec trois unités de production, un laboratoire de reproduction et une unité de commercialisation d'alevins. La base du raisonnement est la basse consommation de poisson au Brésil, qui pourrait facilement être augmentée par une offre à bas prix, que le groupe peut obtenir grâce aux atouts dont il dispose.

Figure $n^{\circ} 11$ Agriculture irriguée et pisciculture

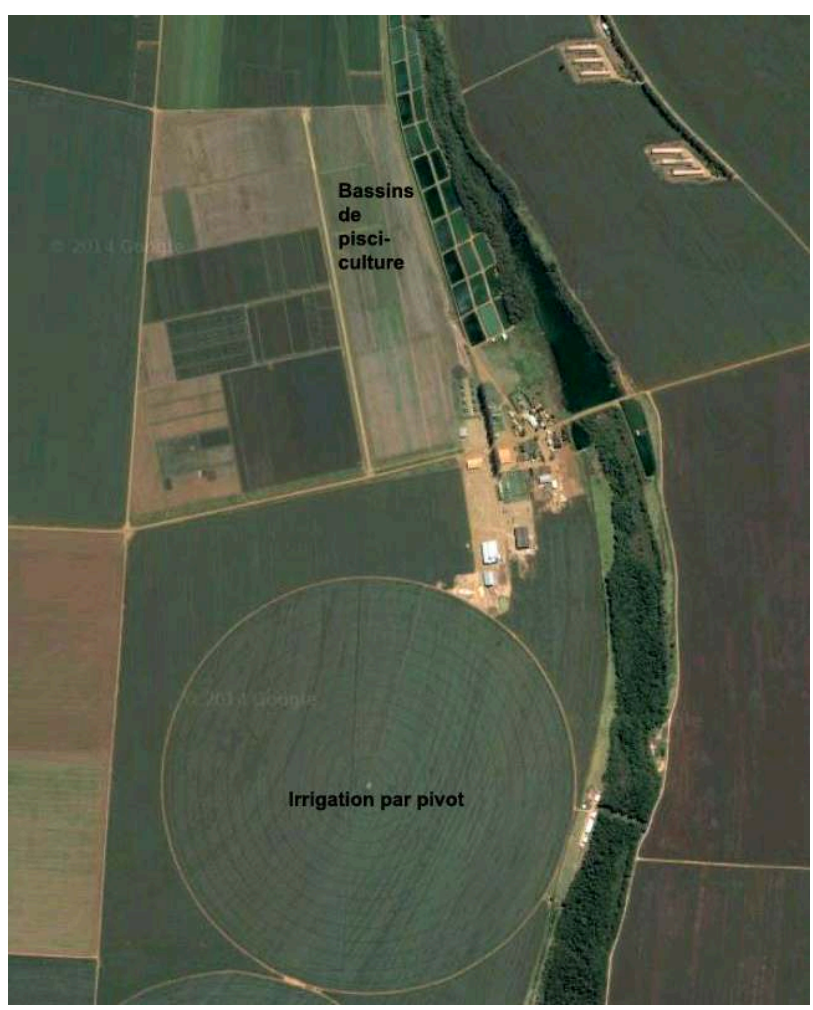

17 Les bassins de production ont été installés sur des sols de moindre qualité, mais où l'on peut disposer de beaucoup d'eau. Pour nourrir les poissons on utilise des sous-produits de l'exploitations agricole, les protéines animales, étant obtenue par le recyclage des déchets de la production piscicole elle-même (têtes et arrêtes, chairs non utilisées par la production de filets de poisson). Au total l'exploitation compte 150 hectares de bassins, avec un rendement d'environ 11 tonnes par ha/an. Les espèces élevées sont des espèces amazoniennes ou du Pantanal (pintado, piau, matrinxã e peraputanga) et une espèce exotique, le tilápia. 


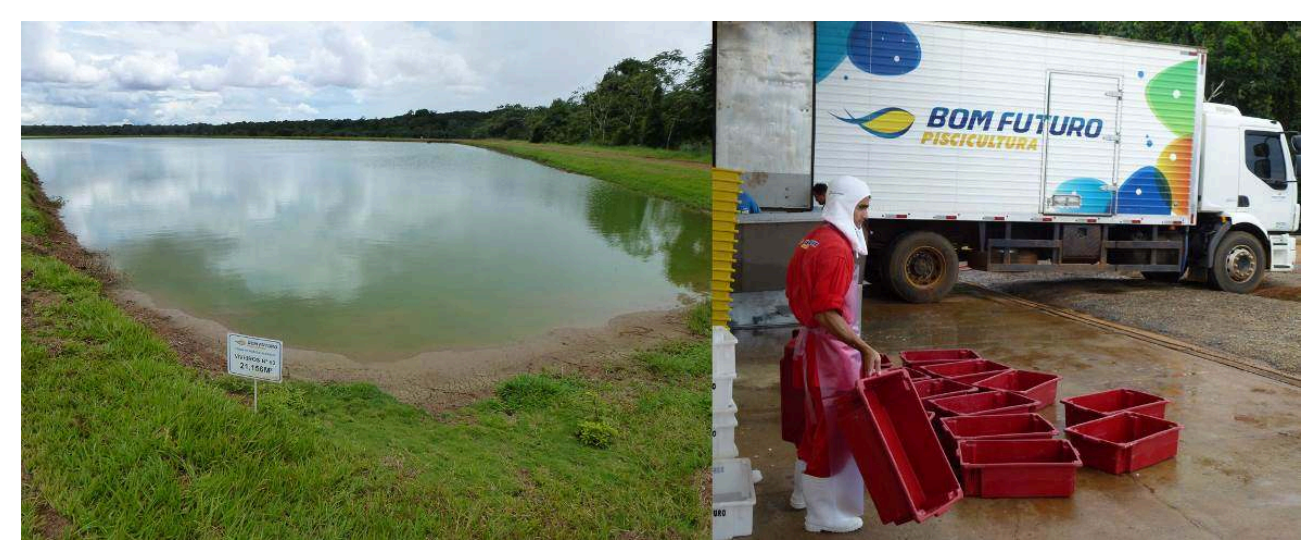

\section{Élevage}

18 Le groupe développe l'élevage bovin depuis 1998, et compte en moyenne 40000 têtes, qui font partie d'un système agriculture-élevage où l'élevage s'intègre au système de rotation des cultures, contribue à la fertilisation des sols (ce qui paraît évident aux agriculteurs européens mais est une nouveauté au Brésil ou élevage et agriculture sont dans l'immense majorité des cas rigoureusement séparés). Les responsables de ce secteur insistent sur le fait que leur activité ne nuit pas à l'environnement par l'ouverture de nouvelles zones dans les parties forestières, mais se cantonne strictement aux zones déjà défrichées.

19 Pour la nourriture du bétail sont utilisés des produits et sous- produits de l'agriculture, notamment du maïs et des tourteaux de soja et de coton. Il s'agit d'une embouche, des lots de bétail maigre sont engraissés en confinement, un lot de mai à juillet, un autre de septembre à novembre, suivis par un arrêt au moment des pluies. Le groupe a choisi de participer au système de traçabilité Sisbov garantissant la provenance et de la qualité de la viande par le suivi des bovins, on distingue sur la photo ci-dessous les boucles d'identification jaunes rivetées dans l'oreille des bêtes, qui permettent de les suivre de la naissance à l'abattoir. 


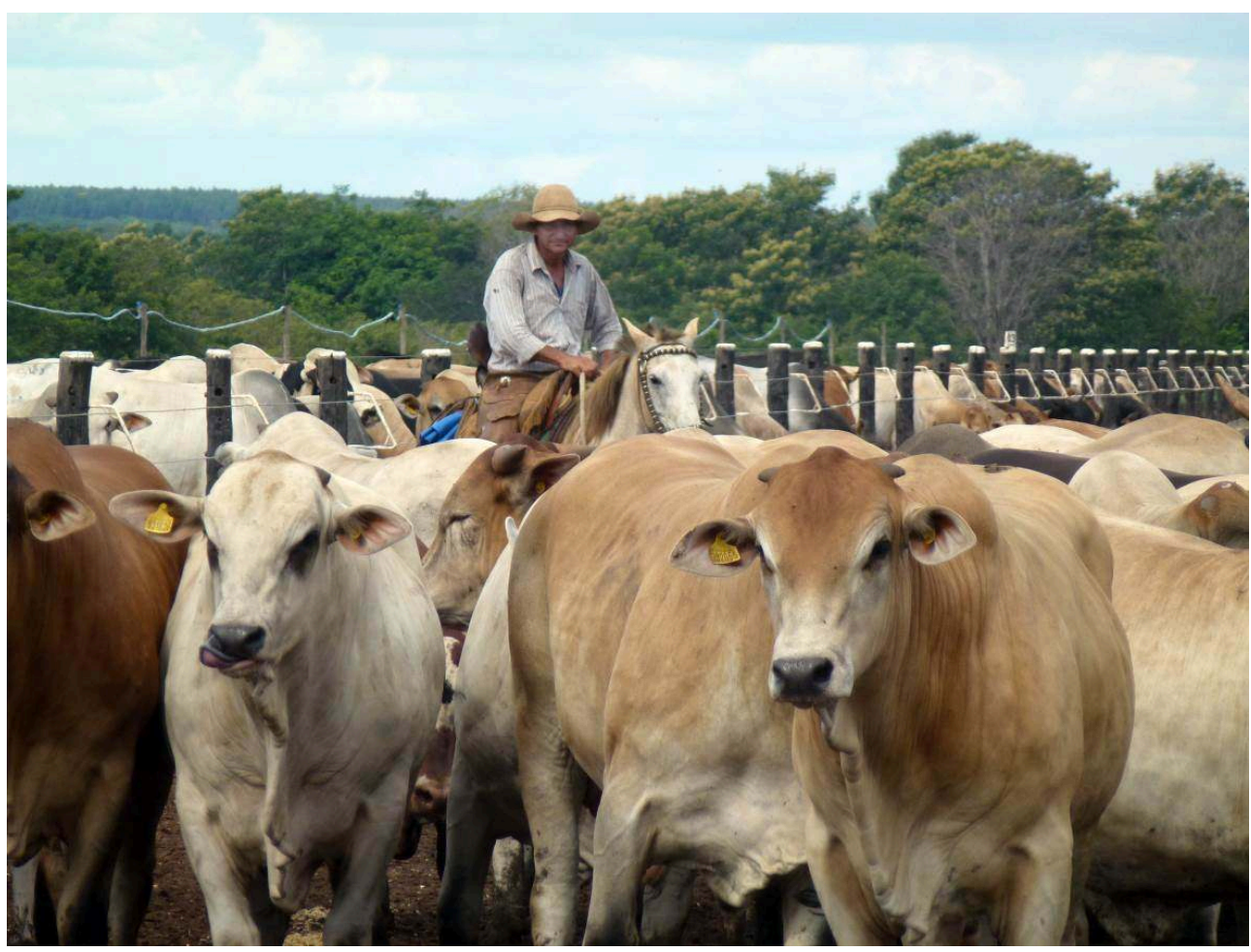

\section{Transports et énergie} ceux du transport de leurs produits vers les marchés consommateurs et la fourniture d'énergie. Le plus grave est sans conteste l'écoulement de leur récolte, en raison de la distance entre les zones de production et le principal port exportateur, Paranaguá, dans le Paraná. Ce port avait été choisi et équipé pour exporter le soja brésilien (et paraguayen) du temps où l'essentiel de la production se faisait dans le Sud, une décision alors logique mais qui est devenue de plus en plus absurde à mesure que les zones productrices se sont déplacées vers le nord.

Les distances à parcourir sont aujourd'hui démesurées, surtout si on les juge à l'aune européenne. Pour mieux apprécier les kilométrages que les camions de soja doivent parcourir, pour atteindre le port de Paranaguá depuis quelques-unes des villes de la région du soja, on les a rapprochées de distances similaires calculées depuis Paris (dans les deux cas en utilisant des logiciels de calcul de distances routières). Campos Novos dos Parecis est à 2136 kilomètres du port d'embarquement, soit la distance ParisTirana (Albanie) ou Paris-Minsk (Biélorussie). Sorriso est à 2196 kilomètres, soit ParisSkopje (Macédoine), Sinop à 2290 kilomètres, soit Paris-Bergen (Norvège), Alta Floresta à 2603 kilomètres, soit Paris - Istanboul (Turquie).

22 C'est pourquoi des solutions alternatives commencent à se mettre en place. Des lignes de chemin de fer ont été construites ou réformées pour atteindre les zones de production, ou du moins s'en rapprocher : elles arrivent actuellement á Rondonópolis, dans le sud du Mato Grosso. Et un port céréalier a été construit par le groupe Maggi à Itacoatiara, sur l'Amazone (un peu en aval de Manaus), desservi par des barges fluviales 
descendant le rio Madeira au départ de Porto Velho (Rondônia). De là le soja part vers les marchés européens et asiatiques par navires de haute mer, d'une capacité volontairement limitée à 55000 tonnes (navires dits Panamax) : quand ils descendent l'Amazone, puis font cap vers le nord, ils ne savent pas encore quelle sera leur destination finale, Europe ou Asie, et il faut donc qu'ils puissent, si l'ordre vient d'aller vers la Chine - le client principal - passer par le canal de Panama. Ces voies nouvelles offrent donc des alternatives, mais même pour atteindre le terminal de Porto Velho les distances restent grandes, et peuvent être à peu près équivalentes pour les producteurs du sud du Mato Grosso, dans le cas de Campos Verde $1580 \mathrm{~km}$ contre 1720 pour Paranaguá.

Pour couvrir la - considérable - consommation électrique du domaine, trois centrales hydroélectriques ont été construites, elles assurent en temps normal les besoins du domaine, et vendent même leur production excédentaire au réseau électrique national. Cependant, les besoins d'énergie pour le séchage des semences et le stockage du grain sur place créent des besoins croissants et l'alimentation électrique par les barrages n'étant plus suffisante, il a fallu installer plusieurs gros générateurs diesel. Pour y remédier, le système de séchage des grains a récemment été changé, en passant du propane à un système à base de bois d'eucalyptus pour alimenter les conduits d'air chaud allant aux bacs de séchage. Le coût du système utilisant le bois d'eucalyptus comme source de chaleur est d'environ $20 \%$ du coût de propane pour la même quantité de séchage.

En guise de conclusion - légèrement ironique - on pourrait dire, en rappelant la célèbre phrase de Vladimir Ilitch Oulianov, dit Lénine(1870-1924), dans son discours au 8e Congrès des Soviets, 1919, "Le communisme, c'est les Soviets, plus l'électricité » qu'avec cette production électrique le groupe Bom Futuro se qualifie encore plus - il en sera sans doute surpris - comme digne héritier des sovkhozes soviétiques.

Le côté soviétique est évidemment ce qui fait la différence entre eux et cette entreprise vigoureusement capitaliste. Mais on rappellera que la taille moyenne d'un sovkhoze était de 15300 hectare (près de trois fois celle des kolkhozes, 5900 hectares en 1990) et que les sovkhozes étaient plus nombreux en Asie centrale, les partie pionnières de l'Union soviétique, que dans sa partie européenne, comme ces exploitations géantes sont plus fréquentes sur les marges pionnières du Brésil que dans ses parties centrales. Ces terres agricoles collectives représentaient en $199024 \%$ du territoire russe, soit 406 millions d'hectares, mais la Russie a définitivement mis fin en 2002 aux kolkhozes et sovkhozes. Une large partie de l'opinion publique étant hostile à leur privatisation, il a fallu plusieurs semaines de négociations au président Vladimir Poutine pour parvenir à un compromis, et le gouvernement espère ainsi accélérer l'arrivée de nouveaux capitaux pour moderniser une agriculture arriérée où la productivité reste très faible. Qui sait si les agriculteurs brésiliens du genre du groupe Bom Futuro ne pourraient pas s'y intéresser, si l'agriculture était ouverte aux étrangers - ce qui n'est pas le cas actuellement - voire s'ils étaient appelés á apporter leur aide comme ils l'ont été au Mozambique.

En attendant, il reste à espérer que la présentation de colosse agro-industriel serve à porter de nouveaux éléments sur l'agora des discussions en cours sur l'avenir de l'agriculture brésilienne, et la concurrence qu'elle fait aux agricultures européennes. 


\section{NOTES}

1. Une vidéo institutionnelle de $15 \mathrm{mn}$ est disponible sur le site du groupe http:// www.bomfuturo.com.br/

\section{RÉSUMÉS}

Une visite du groupe Bom Futuro, situé à Campo Verde, à une centaine de kilomètres à l'Est de Cuiabá apporte quelques éléments concrets pour faire comprendre l'immensité de ces nouvelles exploitations agricoles. Le groupe cultive 420000 ha (dont 230000 ha de soja, 105000 ha de maïs, 75000 ha de coton) en deux récoltes, sur 250000 ha cultivables, et le domaine compte en outre 160000 ha de terres destinées à l'élevage et aux réserves forestières.

Uma visita ao Grupo Bom Futuro, situado a cem quilômetros a leste de Cuiabá em Campo Verde, traz alguns elementos concretos para compreendermos a imensidão das novas explorações agrícolas. O Grupo cultiva 420000 ha (sendo 230000 ha de soja, 105000 ha de milho e 75000 ha de algodão) em duas colheitas em 250.000 há cultiváveis, além de 160000 ha de terras destinadas ao gado e às reservas florestais.

A visit to the Bom Futuro Group, located in Campo Verde, a hundred kilometers east of Cuiabá provides some concrete evidence to understand the immensity of these new farms. The group cultivates 420.000 hectares (including 230.000 ha of soybeans, 105.000 ha of maize, 75.000 hectares of cotton) in two crops on 250.000 ha arable and has 160.000 ha of land for livestock and forest reserves.

Una visita al Grupo Bom Futuro, situado a unos cien kilómetros al este de Cuiabá en Campo Verde, trae algunos elementos concretos para la comprensión de la inmensidad de las nuevas explotaciones agrícolas. El grupo cultiva 420000 hectáreas (siendo 230000 ha de soja, 105000 del maíz y 75000 ha de algodón) en dos colectas, en 250.000 hectáreas en producción, además de 160000 hectáreas de tierra destinado a bovinos y bosque de reservas.

\section{INDEX}

Palabras claves : agri-business, Grupo Bom Futuro, Mato Grosso, tecnología, nuevas explotaciones

Palavras-chave : agro-negócio, Grupo Bom Futuro, Mato Grosso, tecnologia, novas explorações agrícolas

Keywords : agribusiness, Bom Futuro Group, Mato Grosso, technique, new agricultural explorations

Mots-clés : agro-business, Groupe Bom Futuro, Mato Grosso, téchnique, nouvelles exploitations agricoles 
AUTEUR

HERVÉ THÉRY

CNRS-Credal

Professor convidado na USP, Cátedra Pierre Monbeig

hthery@aol.com 